\title{
Preface for a special issue on "Smart environments and collective computational intelligence for disaster management"
}

\author{
Nik Bessis • Eleana Asimakopoulou
}

Published online: 29 December 2012

(c) Springer-Verlag Berlin Heidelberg 2012

Stakeholders in disaster management settings often find the effective and efficient utilisation of emerging technologies quite a challenging process but very frequently a critical computational inclusion to the intelligence required for protecting individuals, communities, their environment and critical infrastructures.

The special issue is dedicated to the dissemination of original contributions that are related to the theories, practices and concepts of smart environments and emerging collective computational and collaborative technologies for the specific purpose of improving intelligence when managing natural or man-made disasters. In particular, the special issue is focused on the applicability-for the purpose of managing disasters - of technologies that can support autonomous adaptation to complex and messy situations caused from these, highly dynamic contexts.

Various methods and technologies including simulations, multi-dimensional decision modeling, data mining, swarm intelligence, smart spaces and sensors, contextaware, situated and pervasive computing, geographical information systems, ad-hoc mobile networks, wireless communications, grid and cloud computing, social networks and crowd sourcing are a smart technology means to enable a more informed decision making to mitigate and prepare for, respond to and recover from growing occurrences of disasters.

This special issue comprises six articles, which have been selected after a careful review process. Specifically,

\footnotetext{
N. Bessis $(\bowtie) \cdot$ E. Asimakopoulou

School of Computing and Mathematics, University of Derby, Kedleston Road, Derby DE22 1GB, UK

e-mail: n.bessis@derby.ac.uk

E. Asimakopoulou

e-mail: eleana.asimakopoulou@googlemail.com
}

this is a collection of extended versions of papers presented either at the Smart Environments for Disaster Management (SEDM-2011) workshop which run in conjunction with the 6th 3PGCIC-2011, held on October 26-28, 2011 in Barcelona, Spain or at the Computational Intelligence for Disaster Management (CIDM-2011) workshop which run in conjunction with the 3rd INCoS 2011, held on November 30-December 2, 2011 in Fukuoka, Japan.

In the first article, Li explains that mobility is an important issue that deters people living in remote areas and elderlies to visit health clinics on a regular basis; this is also the case for individuals with disabilities. Advances in information and telecommunication technologies have been made telemedicine possible. By using the latest sensor technologies, a person's vital data can be collected in a smart home environment. The bio-information can then be transferred wirelessly or via the Internet to medical databases and the healthcare professionals. The article surveys and compiles the state-of-the-art smart home technologies and telemedicine systems.

In the second article, Sakanushi et al. discuss concerns with triage tags, which are widely used at disaster scenes. Some of these concerns include the inability to show the current priorities of casualties and a failure to collect the physiological conditions of the casualties. In their article, authors propose an electronic triage which continuously monitor the vital signs of casualties and transmit them to an electronic triage server. Experimental results show that their proposed electronic triage system can save more lives than the paper triage tags version.

In the third article, Furey et al. explain that the use of Wi-Fi signals is an attractive and reasonably affordable option to deal with the currently unsolved problem of widespread tracking in an indoor environment. In their article, authors present a system which aims at overcoming 
weaknesses in existing real time location systems (RTLS) by using the human approach of making educated guesses about future location. The hypothesis of their work is that knowledge of a person's historical movement habits allows for future location predictions to be made in the short, medium and long term. Their conclusion is that their system improves on the standard Ekahau RTLS in terms of accuracy, latency, cost and prediction.

In the fourth article, Reina et al. highlight that ad hoc networks have been proved to be suitable for disaster scenarios since any infrastructure needs to be deployed in order to establish a wireless network. Authors explain that while one paramount parameter of ad hoc networks is the mobility of nodes, little effort has been made to evaluate the performance of mobile ad hoc networks under mobility models where the movements of rescue teams during evacuating operations are modelled. The objective of their article is to evaluate real case disaster scenarios in terms of performance using several well-known routing protocols metrics.

In the fifth article, Bacon et al. concern with that the main approach to training strategic planners for crisis management (Gold Commanders) continues to be a paperbased. Within this context, authors describe the Pandora Project which has developed an advanced smart environment for the training of Gold Commanders by using artificial intelligence planning techniques to provide a crisis scenario modelled as an event network. Their system includes points of decision for trainees managed by automated rules from a knowledge base, behavioural modelling of the trainees, and ambient management of the environment to provide affective inputs to control and manage trainee stress.

In the sixth article, Silva et al. explain that the simulation of disaster preparedness and mitigation activities requires integration of large amount of data from several distributed data sources. Herein, authors provide an effective way, by the means of the Linked Open Data (LOD) technology so they can bring together distributed data into a standardized and exchangeable common data format. Authors describe the Sahana disaster management system which has been enhanced by the inclusion of this work. Sahana Asia can simulate disaster mitigation and preparedness activities, benefiting vulnerable people, emergency management teams as well as other organizations involved in disaster management. It has been evaluated by participants with some backgrounds in disaster management and found to be effective in terms of system quality, information quality and overall system performance.

At last, the Guest co-Editors would like to thank all the authors of this special issue for their efforts towards the preparation of their manuscripts and for their valuable contributions. We also express our deepest gratitude to the referees for their constructive reviews and commentary. Finally, our sincere thanks go to the Professor Vincenzo Loia, the Editor-in-Chief of the Journal of Ambient Intelligence and Humanized Computing for his excellent support which effectively led to the publication of this special issue. We wish all readers found this issue useful in their research activity. 